\title{
The effect on choice behavior of cues paired with noncontingent reward'
}

RICHARD L, PATTEN AND JERRY W. RUDY

UNIVERSITY OF RICHMOND

The effect of cues paired with reward on the first-trial choice behavior of rats in a Y-maze was examined using the techniques of noncontingent reward in the presence of goal (GB) cues. Adequate perception of GB cues on the test trial was assured by using relatively short maze arms. Significant choice of the arm leading to GB cues was interpreted as suggesting an acquired motivation interpretation of the effect on behavior of cues paired with reward.

In a brilliant series of papers appearing early in the Psychological Review, Clark Hull developed the possibility of accounting for "purposive," "goal-directed," or "instrumental" behavior in terms of underlying (mediating) classical conditioning (e.g., Hull, 1930). A good deal of contemporary behavior theory can be described as "conditioning-model" theory, applying elaborations of the original Hullian ideas to "instrumental" behavior in situations involving reward, nonreward, and aversive stimulation (Amsel, 1965). For example, part of the incentive-motivation theory of Spence $(1956,1960)$ holds that conditionable components $\left(r_{g}\right)$ of the consummatory response to reward become classically conditioned to goalbox (GB) cues. When $r_{g}$ is elicited via stimulus generalization, early in a maze, ongoing performance (locomotion and/or turning) is motivationally facilitated.

Recent experiments (Marx \& Murphy, 1961; Bower \& Grusec, 1964; Trapold, 1966) provide strong evidence for the importance of classical conditioning in interpreting appetitive "instrumental" behavior. Marx \& Murphy (1961) paired a distinctive cue with reward after $S$ had run down a straight runway to the GB. Runway locomotion was facilitated on test trials when the distinctive cue was presented while $S$ was in the startbox. Bower \& Grusec (1964) first trained rats to bar-press and then showed that the prior occurrence of relevant stimuli in a classical conditioning paradigm (noncontingent reward and nonreward) strongly facilitated learning a successive discrimination (where S responds differentially to successively presented stimuli). Trapold (1964) showed the same effect of interpolated noncontingent reward and nonreward on successive discrimination reversal learning. However, in the latter two studies the possible effects of noncontingent reward are mixed with the possible effects of noncontingent nonreward.

The present study was addressed to two general problems: (1) A demonstration of the effectiveness of noncontingent reward on simultaneous discrimination (where $\mathrm{S}$ responds differentially to two or more simultaneously presented stimuli). The present experiment utilized a Y-maze to examine this problem. (2) The appropriateness of a motivational interpretation of the effect of noncontingent reward on choice behavior in the Y-maze. An important difference between motivational and learning facilitation of behavior lies in the immediacy of the behavior enhancement. In addition, a motivational effect is generally regarded as one not attributable to cue-response associative connections. Thus, immediate facilitation of a response (e.g., choice) upon the occurrence of a cue not previously associated with the reference response would be interpretable as a motivational effect.

The possible development of association between the turning component of the choice response and GB cues or response $\left(r_{g}\right)$-produced cues $\left(s_{g}\right)$ was controlled in the present study by using the noncontingent reward technique for GB cue-reward pairings. Ss' first exposure to the choice section of the maze occurred on a single test trial following the noncontingent reward phase of the experiment. The possible development of association between the locomotor component of the choice response and GB cues or $s_{g}$ was assessed by comparing the percent choice performance of two groups of Ss. Group DP Ss were given noncontingent reward by placement directly over the food dish in a GB small enough to prevent locomotion toward the food. Group DPR Ss were placed in a longer $G B$ in a manner that required $S$ to locomote $8 \mathrm{in}$. to get to the food dish.

Method

The Ss were 42 naive male Long-Evans hooded rats 90 days old at the start of the experiment. The apparatus was a Y-maze with a stem 18 in. long and arms 10 in. long. The floor of the maze was 4 in. wide and the maze sides were 4 in. high. The entire maze was covered with hinged Plexiglas and was painted a flat black except for the removable lining of the GB. The floor, sides and end of the $10 \mathrm{in}$.long GB was lined with white paper sprinkled liberally with 1/4-in. black polka dots. A 6-volt DC light was mounted 3 in. above the food dish at the end of the GB. The experimental room of dimensions $6 \mathrm{ft}$. $\times 6 \mathrm{ft}$. with a ceiling height of $7-1 / 2 \mathrm{ft}$. was lighted by a 15-watt incandescent bulb located 55 in. above the maze stem. Ss were handled 2-5 min. per day for the eight days prior to the noncontingent reward training phase of the experiment and were put on a fixed-amount food maintenance regimen of $9 \mathrm{gm}$ of Purina lab pellets per day for five days prior to experimentation and during the experiment.

Ss were divided into three groups of 14 Ss each. During the noncontingent reward phase all groups of Ss were given four direct placements in the GB per day for six days. Daily trials were separated by an 8-10 min. interval. During this phase the GB lining was 
located in the last $10 \mathrm{in.} \mathrm{of} \mathrm{the} \mathrm{maze} \mathrm{stem.} \mathrm{Each} \mathrm{DP} \mathrm{S}$ was placed in the 10 in. GB with his nose directly over the food cup. For DPR Ss the GB was lengthened to $18 \mathrm{in.}$ and $\mathrm{S}$ was required to locomote $8 \mathrm{in}$. to the food cup. Ss in a third, control, group of Ss (group C) were treated like DPR Ss with the exception that no food was in the food cup on their direct placement trials. Group C Ss were placed into a foodless 18-in. GB for a duration equal to the mean time taken by DP and DPR Ss to consume their food on that trial. DP and DPR Ss received $3 \mathrm{gm}$ of wet mash per direct placement, which was consumed in about $60 \mathrm{sec}$. The $42 \mathrm{Ss}$ were run in two squads of 21 Ss each. Ss in each squad were fed in their home cages immediately after being run, with DP and DPR Ss getting 6 gm of pellets and C Ss getting $9 \mathrm{gm}$ of pellets. The total weight of dry food given to each $\mathrm{S}$ in the three groups was $9 \mathrm{gm}$ per day. Water was continuously available in the home cages.

After a single direct placement trial on day 7 of the experiment the GB lining, 6-volt DC light, and a clean food dish were placed in the left arm of the maze. Dishes of wet mash were placed in both maze arms for $30 \mathrm{~min}$. to equate olfactory cues in the arms and then removed, after which each $\mathrm{S}$ was given a single choice test trial. A choice response was defined by $\mathrm{S}$ breaking a photocell beam located 8 in. from the end of each arm. Choice time, defined as the time between being placed in the stem and breaking a photocell beam, was recorded for all Ss.

\section{Results and Discussion}

Ss quickly adjusted to the direct placement procedure. After the first day no $S$ from any group urinated or defecated in the GB. On the single test trial 11 DP Ss, $12 \mathrm{DPR} \mathrm{Ss}$, and $2 \mathrm{C}$ Ss chose the GB maze arm. The difference in percent choice between the $28 \mathrm{DP}$ and DPR Ss and the $14 \mathrm{C}$ Ss was significant $(t=4.25, p<.001)$. Homogeneity of choice time variances for the three groups of Ss was ascertained by an F-max. test. Comparing the choice time behavior of Ss choosing the GB arm produced a significant difference between DP and DPR Ss $(t=2.04, p<.05)$, with DPR Ss making the speediest choices. These results are consistent with a motivational interpretation of the effect on "instrumental" behavior of cues paired with reward (e.g., Spence, 1956), as well as with the writers' previous observation and interpretation of the acquisition of orienting behavior toward the conditioning stimulus during classical conditioning of the licking response in rats. ${ }^{2}$ The fact that DP and DPR Ss differed on choice time but not on percent choice might be taken as suggesting the possibility that, though notessential, associative factors may contribute to the determination of choice behavior. However, the DP-DPR choice time difference may be due to differences between these groups in the similarity of GB cues as they appeared during the training phase and as they appeared when viewed from the choice point on the test trial. There would be greater training-test similarity for Ss who had to locomote $8 \mathrm{in}$. to the food dish during training. More specifically, in regard to a distinction between learning and motivation on the basis of immediate versus gradual behavior facilitation, the present results support a motivational interpretation of the effect on behavior of cues paired with reward. Though the results of the present study also suggest a nonassociative interpretation of such cue effects, adequate evidence on this matter would require controlling Ss' reward-related associative history from birth until the choice test.

The present experiment is similar in design to a large number of studies that have employed a number of $\mathrm{T}-$ maze choice test trials to GB cues paired with differential reward on straight runway training trials, or to a GB paired with reward and a new GB (e.g., Hall, 1951; D'Amato, 1955; Hopkins, 1955; Klein, 1959; et al). The apparent discrepancy between the strong, immediate effect on choice performance of cues paired with reward obtained in the present study and the gradually increasing facilitation of choice behavior in the secondary reinforcement studies is understandable after inquiry into the method sections of the latter studies reveals the variety of means employed to block out sight of the GB cues from the choice point. E-mazes, U-mazes, closed GB retrace doors, swinging gates, curtains and darkness have all been employed to prevent the direct, immediate effect of GB cues on choice behavior. A simple application of the concepts of higher order classical conditioning and stimulus generalization may be utilized to coordinate the data and language contained in secondary reinforcement studies such as those mentioned above with the data and acquired motivation terminology of the present study. The acquired motivation language appears to hold some promise of being the more generally useful of the two terminologies.

\section{References}

Amsel, A. On inductive versus deductive approaches and neoHullian behaviorism. In B. B. Wolman (Ed.), Scientific psychology. New York: Basic Books, 1965. Pp. 187-206.

Bower, G., \& Grusec, T. Effect of prior Pavlovian discrimination training upon learning an operant discrimination. J. exp. Anal. Behav., 1964, 7, 401-404.

D'Amato, M. R. Secondary reinforcement and magnitude of primary reinforcement. $J$. comp. physiol. Psychol., 1955, 48, 378-380.

Hall, J. F. Secondary reinforcement as a function of the frequency of primary reinforcement. J. comp. psychol., 1951, 44, 246-251.

Hopkins, C. O. Effectiveness of secondary reinforcing stimuli as a function of the quantity and quality of food reinforcement. $J$. exp. Psychol., 1955, 50, 339-342.

Hull, C. L. Knowledge and purpose as habit mechanisms. Psychol. Rev., 1930, 37, 511-525.

Klein, R. M. Intermittent primary reward as a parameter of secondary reinforcement. J. exp. Psychol., 1959, 58, 423-427.

Marx, M. H., \& Murphy, W. W. Resistance to extinction as a function of the presentation of a motivating cue in the startbox. J. comp. physiol. Psychol., 1961, 54, 207-210.

Spence, K. W. Behavior theory and conditioning. New Haven: Yale University Press, 1956.

Spence, K. W. Behavior theory and learning. Englewood Cliffs: Prentice-Hall, 1960.

Trapold, M. A. Reversal of an operant discrimination by noncontingent discrimination reversal training. Psychon. Sci., 1966, 4 , 247-248.

\section{Notes}

1. Supported in part by NIMH grant 1B03MH12569-01 to R. Patten. 2. Paper presented to the Virginia Academy of Science, Harrisonburg, Va., 1966. 